\title{
Minimizing Iridium Oxide Electrodes for High Visual Acuity Subretinal Stimulation
}

\author{
Samir Damle, ${ }^{1}$ Maya Carleton, ${ }^{2}$ Theodoros Kapogianis, ${ }^{2}$ Shaurya Arya, ${ }^{3}$ Melina Cavichini-Corderio, ${ }^{4}$ \\ William R. Freeman, ${ }^{4}$ Yu-Hwa Lo, ${ }^{3}$ and ${ }^{1}$ Nicholas W. Oesch ${ }^{2,4}$
}

\section{https://doi.org/10.1523/ENEURO.0506-20.2021}

${ }^{1}$ Department of Bioengineering, University of California San Diego, La Jolla, CA 92093, ${ }^{2}$ Department of Psychology, University of California San Diego, La Jolla, CA 92093, ${ }^{3}$ Department of Electrical and Computer Engineering, University of California San Diego, La Jolla, CA 92161, and ${ }^{4}$ Jacobs Retina Center at Shiley Eye Institute, Department of Ophthalmology, University of California San Diego, La Jolla, CA 92093

\begin{abstract}
Vision loss from diseases of the outer retina, such as age-related macular degeneration, is among the leading causes of irreversible blindness in the world today. The goal of retinal prosthetics is to replace the photo-sensing function of photoreceptors lost in these diseases with optoelectronic hardware to electrically stimulate patterns of retinal activity corresponding to vision. To enable high-resolution retinal prosthetics, the scale of stimulating electrodes must be significantly decreased from current designs; however, this reduces the amount of stimulating current that can be delivered. The efficacy of subretinal stimulation at electrode sizes suitable for high visual acuity retinal prosthesis are not well understood, particularly within the safe charge injection limits of electrode materials. Here, we measure retinal ganglion cell (RGC) responses in a mouse model of blindness to evaluate the stimulation efficacy of 10,20 , and $30 \mu \mathrm{m}$ diameter iridium oxide electrodes within the electrode charge injection limits, focusing on measures of charge threshold and dynamic range. Stimulation thresholds were lower for smaller electrodes, but larger electrodes could elicit a greater dynamic range of spikes and recruited more ganglion cells within charge injection limits. These findings suggest a practical lower limit for planar electrode size and indicate strategies for maximizing stimulation thresholds and dynamic range.
\end{abstract}

Key words: blindness; electrical stimulation; neural stimulation; prosthetic; retina; retinal prosthetic

\section{Significance Statement}

Neural prosthetics offer hope to cure intractable neurologic disorders. To enable fine control over patterns of neural activity, stimulating electrode size must be decreased to the scale of neurons. We examined how electrode size at this scale influences stimulation threshold and the range of possible responses, by fabricating planar iridium oxide electrodes between 10 and $30 \mu \mathrm{m}$ in diameter, and examined neural stimulation in a mouse model of retinal degeneration. This work provides new insights into how small stimulation electrodes translate charge into neural activity and the physical factors that contribute to differences between responses over this range of electrode sizes. This has important implications for the design of high-acuity retinal prosthetics, as well as next-generation neural stimulators.

\footnotetext{
Received November 23, 2020; accepted November 15, 2021; First published November 19, 2021.

S.D., Y.-H.L., W.R.F., and N.W.O. have an equity interest in Nanovision Biosciences, Inc. Y.-H.L., W.R.F., and N.W.O. also serve on the Scientific Advisory Board. Although this grant (EY029259) has been identified for conflict of interest management based on the overall scope of the project and its potential benefit to Nanovision Biosciences, Inc., the research findings included in this particular publication may not necessarily relate to the interests of Nanovision Biosciences, Inc. The terms of this arrangement have been reviewed and approved by the University of California, San Diego in accordance with its conflict of interest policies. All other authors declare no competing financial interests.
}

\section{Introduction}

The number of patients with sight-threatening retinal diseases is increasing (Wong et al., 2014; Bourne et al., 2017). Dystrophic or degenerative retinal diseases result in the progressive loss of photoreceptors, causing vision

Author contributions: S.D. and N.W.O. designed research; S.D., M.C., and T.K., S.A., performed research; S.D., M.C., T.K., S.A., and N.W.O. analyzed data; S.D., W.R.F., Y.-H.L., and N.W.O. wrote the paper. 
loss (Hartong et al., 2006; Jones et al., 2016; Pennington and DeAngelis, 2016). Currently there are limited options to slow progression, and no options to recover lost vision. Early retinal prostheses have demonstrated promising prosthetic visual acuity results between 20/500 and 20/ 1520 in clinical trials (Humayun et al., 2012; Stingl et al., 2015; Palanker et al., 2020). To improve on the visual benefits provided by retinal prostheses, recent research efforts have focused on developing implants with more densely packed microelectrodes to support higher visual acuity (Lorach et al., 2015; Ha et al., 2016; Damle et al., 2020).

While different retinal implant technologies have been developed to translate an image into a spatial pattern of electrical stimulation, all deliver electric charge through electrodes to stimulate action potentials in retinal ganglion cells (RGCs), the output neurons of the retina. RGCs can be directly activated by electrical stimulation or indirectly through synaptic input when upstream neurons, such as retinal bipolar cells are excited. The ultimate goal of retinal prosthetics is to create patterns of action potentials in the RGC layer that encode the spatiotemporal properties of visual scenes, which are then relayed to the brain (Goetz and Palanker, 2016; Yue et al., 2016).

The limit of prosthetic spatial resolution is determined by the size and spacing of stimulating electrodes in implanted arrays (Palanker et al., 2005; Wilke et al., 2011). To increase spatial resolution, there is significant pressure to decrease electrode size; however, there is a trade-off between electrode size and the amount of charge that can be safely injected, termed the charge injection capacity (CIC; Ganji et al., 2017). Beyond this limit, irreversible damage to both neural tissue and electrode materials occur (Cogan, 2008). Retinal implants currently in clinical testing use planar disk shaped electrodes, ranging from 30 to $200 \mu \mathrm{m}$ diameters and are composed of sputtered iridium oxide film (SIROF), an electrode material with high CIC (Cogan et al., 2009; Shire et al., 2009; Boinagrov et al., 2016; Ha et al., 2016; Yang et al., 2016).

Electrical stimulation of retinal cells has been studied extensively with ex vivo retinal preparations using a wide variety of electrode materials and geometry (Stett et al., 2000; Yang et al., 2011, 2018; Cai et al., 2013; Sim et al., 2014; Im and Fried, 2015; Stutzki et al., 2016). These studies have provided valuable insight into electrical requirements for some electrode configurations; however, there is still considerable variability in the results reported, and much less attention to the range of possible responses beyond the stimulation threshold, particularly within the practical limits of safe injectable charge. As a result, there is little consensus of ground truth principals

This work was supported by the National Institutes of Health Grant EY029259 (to N.W.O.).

Correspondence should be addressed to Nicholas W. Oesch at noesch@ ucsd.edu.

https://doi.org/10.1523/ENEURO.0506-20.2021

Copyright (C) 2021 Damle et al.

This is an open-access article distributed under the terms of the Creative Commons Attribution 4.0 International license, which permits unrestricted use, distribution and reproduction in any medium provided that the original work is properly attributed. that establish a framework for electrode development for high visual acuity subretinal prostheses.

Here, we address these questions by examining retinal stimulation elicited by planar microelectrodes of 10, 20 , and $30 \mu \mathrm{m}$ diameter. We first established the charge limits of these electrodes, to establish the realistic bounds of maximal stimulation. We then varied current magnitude and pulse width within these limits to characterize the stimulation threshold and dynamic range of RGC spiking at each electrode diameter, both important measures of efficacy. We found that smaller electrodes have lower charge thresholds, but recruited fewer RGS and have a reduced dynamic range compared with larger electrodes. Importantly, these results demonstrate that charge density alone does not normalize efficacy of stimulation across different electrode diameters, but electric field modeling shows that the spatial extent of the electric field may be important when determining stimulation efficiency even at the same charge density. Since electric field size is influenced by both electrode size and stimulation charge, this finding places constraints on neural prosthetic electrode design. Taken together, this work establishes a framework to develop and compare new microelectrodes for high visual acuity prosthesis within realistic limits.

\section{Materials and Methods}

\section{Microelectrode fabrication}

Iridium oxide electrodes were patterned by photolithography. Transparent conductive traces of indium tin oxide (ITO) were deposited on borosilicate glass by RF magnetron sputtering and were insulated by a $200 \mathrm{~nm}$ layer of SiNx deposited by plasma-enhanced chemical vapor deposition (PECVD). Iridium oxide electrodes of 10, 20 , and $30 \mu \mathrm{m}$ diameter stimulating electrodes and $400-\mu \mathrm{m}$ return electrodes were formed by reactive DC sputtering of iridium metal in an argon (90\%) and oxygen (10\%) gas mixture at a thickness of $600 \mathrm{~nm}$. Electrodes were spaced $50 \mu \mathrm{m}$ apart, from electrode center to center. Electrode arrays consisted of four rows of eight electrodes, with the top two rows consisting of $10 \mu \mathrm{m}$ diameter electrodes, the bottom left quadrant of electrodes consisting of 20 $\mu \mathrm{m}$ diameter electrodes, and the bottom right quadrant consisting of $30 \mu \mathrm{m}$ diameter electrode. (Fig. 1). Finally, a $1.5 \mu \mathrm{m}$ insulating layer of parylene-C was deposited over the electrode array (PDS 2010 Coater) and iridium oxide electrodes were exposed by oxygen plasma reactive ion etching (RIE) etching (Oxford Plasmalab 80; Fig. 1)

\section{Electrochemical characterization}

Electrochemical characterization was performed using a three-electrode configuration consisting of the iridium oxide electrode as the working electrode, an $\mathrm{Ag} / \mathrm{AgCl}$ electrode as the reference, and a platinum wire as the counter electrode (Gamry Interface 1000E). Cyclic voltammetry measurements were performed to determine the charge storage capacity (CSC) of the electrodes. The electrode potential was swept cyclically between -0.6 and $0.8 \mathrm{~V}$, relative to the reference electrode at constant 


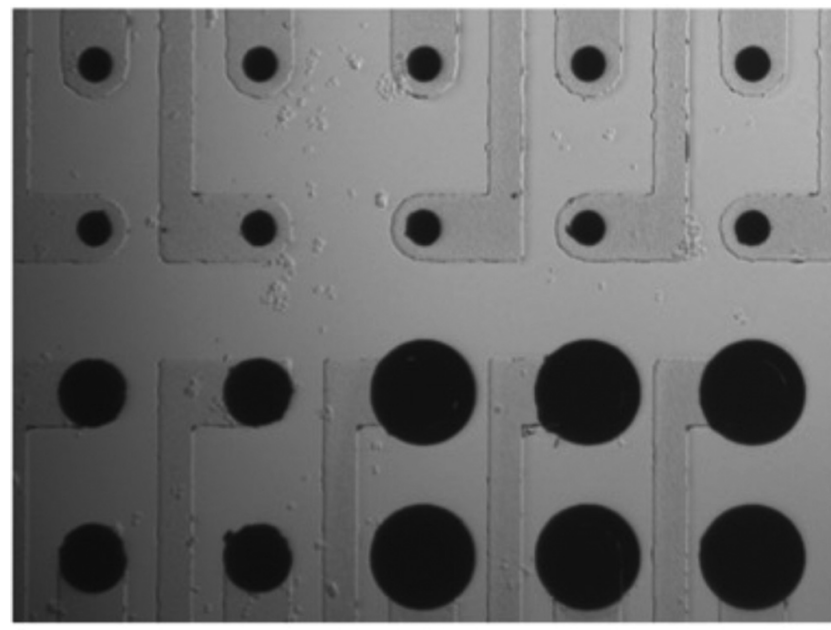

Figure 1. Image of a region of the microfabricated array of sputtered iridium oxide electrodes of $10 \mu \mathrm{m}$ (top), $20 \mu \mathrm{m}$ (bottom left), and $30 \mu \mathrm{m}$ (bottom right) diameter electrodes at $50 \mu \mathrm{m}$ pitch.

scan rate of $200 \mathrm{mV} / \mathrm{s}$ at $10 \mathrm{mV}$ increments while measuring the flow of current from the working electrode to the counter electrode. The CSC was calculated using the time integral of the cathodic and anodic currents up to the potential limits of the water window; the voltage range where electrochemical reactions occurring at the electrode-electrolyte interface are reversible.

The CIC of the electrode, defined as the maximum amount of charge that can be delivered without polarizing the electrode potential beyond the water window, was determined by measuring the transient voltage change between the working and reference electrode in response to a square biphasic anodic first current pulse injected between the working and counter electrode, measured as the near-instantaneous voltage change immediately after the current pulse is terminated $\left(E_{m a}\right.$ or $E_{m c}=\Delta V-V_{a}$; Cogan, 2008). The electrode polarization at the anodal and cathodal phase were measured $10 \mu \mathrm{S}$ after the end of each respective phase of the stimulation pulse. All measurements were made in $126 \mathrm{~mm} \mathrm{NaCl}$.

\section{Retina explant and loose patch electrical recording}

All experimental methods and animal care procedures were conducted in accordance with National Institutes of Health guidelines and were approved by the University of California, San Diego Institutional Animal Care and Use Committee. Adult rd1 or rd10 mice, of either sex ( $>$ postnatal day 60 ) with photoreceptor cell degeneration were anesthetized with isoflurane and euthanized by decapitation and their retinas were isolated and maintained in Ames medium oxygenated and equilibrated with $95 \% \mathrm{O}_{2}$, $5 \% \mathrm{CO}_{2}$. Retina pieces, $\sim 2 \times 2 \mathrm{~mm}$, were transferred to a custom recording chamber and placed over stimulating electrodes on the bottom of the custom recording chamber, ganglion cell side up. The chamber was placed under an upright microscope and perfused with Ames solution $(4 \mathrm{ml} / \mathrm{min})$ at $35^{\circ} \mathrm{C}$. Microscopy was used to visualize and confirm contact between the outer portion of the retina and the electrode array surface. RGCs were visualized and targeted using IR differential interference contrast video microscopy. Given that the stimulating electrode pitch is $50 \mu \mathrm{m}$, this means that our recordings are from cells that are within $\sim 25 \mu \mathrm{m}$ of the stimulating electrodes. Furthermore, given our arrangement of the different electrode diameters on the same array, we have several regions, where we can record from cells that are equidistant between different electrode diameters.

Recording electrodes were pulled from borosilicate capillary glass to have a final resistance of 4-5 M $\Omega$ and filled with Ames medium. Loose-patch recordings were made from ganglion cells and action potentials were recorded in voltage-clamp mode using a Multiclamp 700b (Molecular Devices) patch-clamp amplifier. Signals were filtered at $4 \mathrm{kHz}$ (4-pole Bessel), digitized at $20 \mathrm{kHz}$ with an ITC-18 (HEKA Electronik) data acquisition board and saved to a PC for offline analysis using custom acquisition software in IgorPro 7 (WaveMetrics). SIROF electrons were wired to a 32-channel RHS2000 stim and recording system (Intan Technologies). Charge-balanced, anodic first, square biphasic current pulses were generated on the RHS2000, which was triggered by our acquisition software, and delivered to an individual stimulating electrode nearest to the cell of interest. Individual stimulus pulses or pulse trains were delivered every $10 \mathrm{~s}$ and repeated five times. Individual cell responses were calculated from the average of the five repeats. Spontaneous activity was subtracted from stimulation evoked activity by measuring the spontaneous firing rate for $2 \mathrm{~s}$ before the stimulus, and measuring any change in evoked response above this baseline level. To measure threshold, half-max and dynamic range, linear interpolation between stimulation levels was used to estimate the current needed to achieve a particular metric.

\section{Simulation of electric field}

Simulations of electric field were performed using a 3-D finite element model of the electrode array and retina for static current flow inside of a volume conductor using COMSOL Multiphysics 5.2b (COMSOL AB) on a personal computer running Windows 7 . A model of a single stimulating electrode was constructed for 10,20 , and $30 \mu \mathrm{m}$ diameters. The electrode was centered inside of a passive domain consisting of a homogenously conductive $(0.2 \mathrm{~S} /$ $\mathrm{m})$ sphere of $18 \mathrm{~mm}$ diameter (Wang and Weiland, 2015). A single large return electrode of $200 \mu \mathrm{m}$ diameter was positioned at a distance of $7.5 \mathrm{~mm}$ from the center of the electrode array.

The magnitude of the electric field $(E)$ at a particular electrode-cell separation distance is a function of the current density produced at an electrode $(J)$ and the tissue conductivity ( $\sigma$, assumed to be constant). The voltage potential distribution inside of the passive domain was modeled according to the Poisson equation and continuity equation (Wilke et al., 2011):

$$
\nabla \cdot J=\nabla \cdot(-\sigma \nabla \mathrm{V})=0 .
$$



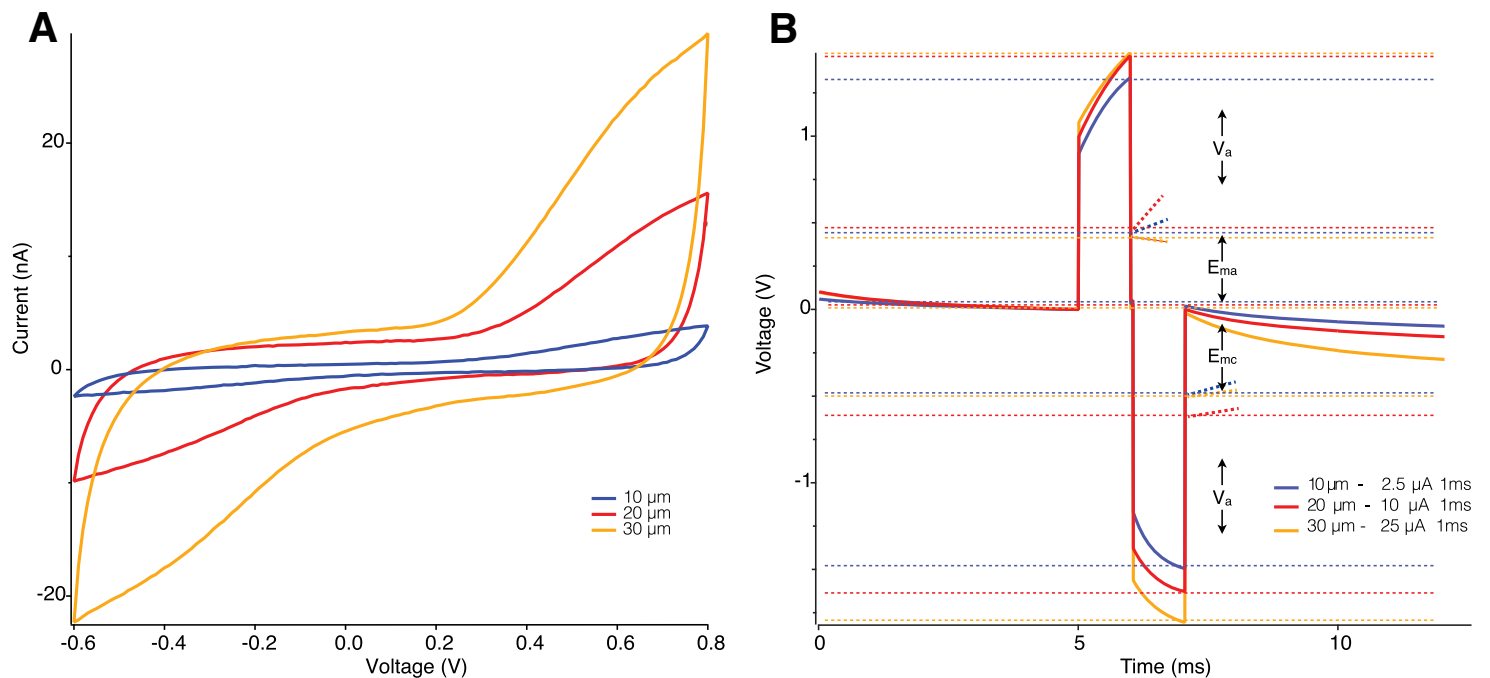

Figure 2. Electrochemical characterization of $10 \mu \mathrm{m}$ (blue), $20 \mu \mathrm{m}$ (red), and $30 \mu \mathrm{m}$ (orange) sputtered iridium oxide electrodes. $\boldsymbol{A}$, Cyclic voltammetry scan used to calculate the electrochemical CSC of the electrodes. $\boldsymbol{B}$, Voltage transients in response to a biphasic current pulse used to calculate the safe $\mathrm{CIC}$ of electrodes within the polarization limits of the water window of iridium oxide. The rapid change in potential represents the voltage drop across the electrolyte solution $\left(V_{a}\right)$ while the gradual change in potential represents the polarization of the electrode $\left(E_{m a}\right.$ and $\left.E_{m c}\right)$. Dashed lines represent the voltage boundaries for $V_{a}$ and $E_{m a}$ and $E_{m c}$ for 10 , 20 , and $30 \mu \mathrm{m}$ electrodes in blue, red, and orange, respectively.

The electric field was determined from the voltage distribution in COMSOL by calculating the gradient of the voltage distribution:

$$
E=-\nabla \mathrm{V}
$$

The distribution of the electric field from the stimulating electrode into the 3-D volume was calculated for a fixed current density set at the surface of each electrode. This model allowed for a simplified electrostatic approach to understand the effect of stimulating current magnitude within safe $\mathrm{CIC}$ limits of each electrode diameter in terms of the magnitude and spatial distribution of the electric field available to induce stimulation of retinal neurons.

\section{Code accessibility}

The code/software described in the paper is freely available online at https://github.com/noeschlab/noeschlab/ tree/noeschlab-ElectrodeSizeCOMSOL. The code is available as COMSOL .mph files in Extended Data 1.

\section{Results}

\section{Electrochemistry}

To examine how electrical stimulation of the retina is influenced by the electrode diameter we fabricated SIROF electrodes at 10,20 , and $30 \mu \mathrm{m}$ diameters. SIROF is widely considered to be the state-of-the-art electrode material for neural prosthetics (Cogan, 2008). These diameters were chosen because they correspond to the size of electrodes that can be packed with an electrode pitch $<50 \mu \mathrm{m}$, the necessary density for a retinal prosthetic array to reach the spatial acuity equivalent of legal blindness (Palanker et al., 2005). Our goal was to explore the range of neural responses that can be realistically stimulated within the electrochemical limits for electrodes of this scale.
We first characterized the CSC of the electrodes at each diameter to ensure their performance was consistent with those typically used in retinal prostheses. CSC provides information about the total amount of electroactive material on the electrode at near-equilibrium conditions. The magnitude of charge that each electrode can deliver scales with the electrode surface area as expected (Fig. 2A). The measured anodal and cathodal charge storage capacities of all electrode diameters were identical at 12 and $17 \mathrm{mC} / \mathrm{cm}^{2}$, respectively. These results fall within the range of previously reported studies of iridium oxide electrodes (Cogan et al., 2009).

Although it has become common practice to characterize electrodes by their CSC, it does not accurately reflect the amount of charge that can be safely delivered per phase during realistic neural stimulation conditions. This property, called the CIC (Cogan, 2008), imposes a limit on the maximum amount of charge that can be used for stimulation without driving the electrode polarization to a level at which irreversible reactions occur that could damage the electrode or tissue. The electrode polarization limit is known as the water window and occurs at $-0.6 \mathrm{~V}$ for cathodal stimulation and $0.8 \mathrm{~V}$ for anodal stimulation for SIROF electrodes. The measured charge injection limit of sputtered iridium oxide electrodes was $3.3 \pm 0.2 \mathrm{mC} / \mathrm{cm}^{2}$, also within the range of previously reported studies of SIROF (Cogan, 2008; Cogan et al., 2009; Haas et al., 2020; Negi et al., 2012). The total safe injectable charge per stimulation pulse scales with area, equaling $2.5,10$, and $25 \mathrm{nC}$ for 10 , 20 , and $30 \mu \mathrm{m}$ electrodes, respectively (Fig. $2 B$ ).

\section{Stimulation evoked ganglion cell responses}

To examine how the retina responds to stimulation by these electrodes we mounted the electrode arrays on the bottom of our standard electrophysiological recording 
A

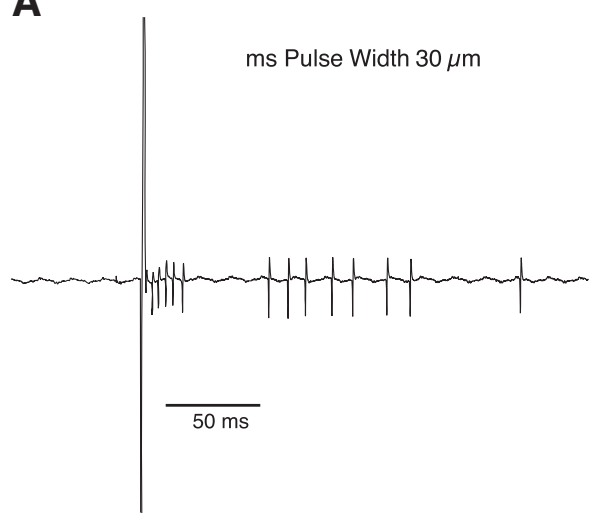

C

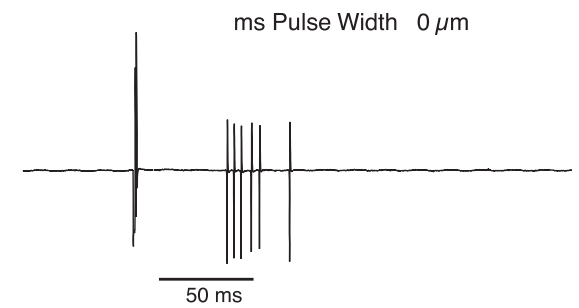

B

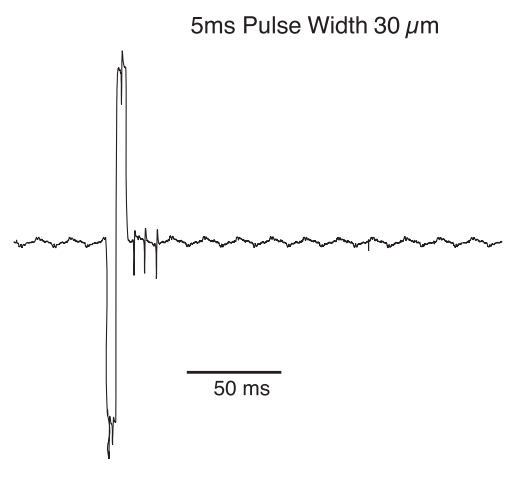

D

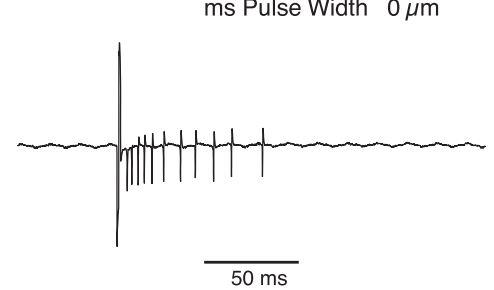

Figure 3. Example spiking responses of RGCs in response to subretinal electrical stimulation with SIROF electrodes measured by loose patch clamp. Stimulation with a $30 \mu \mathrm{m}$ electrode at 1-ms $(\boldsymbol{A})$ or 5 -ms $(\boldsymbol{B})$ pulse width. Stimulation with 1 ms pulse width for a (C) $20 \mu \mathrm{m}$ electrode and (D) $10 \mu \mathrm{m}$ electrode (D).

chamber, and placed the retina outer side down, such that the inner nuclear layer was in contact with the electrode array on the bottom of the chamber and ganglion cells faced up. We used loose-patch recordings to measure ganglion cell spiking from whole mount RD1 or RD10 retina (ages 60-140 d). We did not observe a difference between RD1 and RD10 retinas across a range of stimulation intensities (ANOVA, $p=0.91$ ) and grouped RD1 and RD10 results when both were used.

Given, that we image the electrode array and the RGCs simultaneously, we know the position and distance between the cell of interest and the stimulating electrode. We stimulated cells on the electrode nearest to the cell, when possible, meaning the stimulating electrode was typically $25 \mu \mathrm{m}$ or less from the cell of interest, given our $50 \mu \mathrm{m}$ pitch. We delivered anodic first, biphasic, charged balanced constant current pulses with 1 or 5 ms duration per phase and varied the magnitude of the stimulating current up to the $\mathrm{CIC}$ limit of the electrode. Anodic first pulses have previously been shown to have lower charge thresholds for subretinal stimulation (Boinagrov et al., 2014). Electrical stimulation elicited bursts of action potentials following charge delivery (Fig. 3).

\section{Maximizing retinal response within charge injection limits}

Retinal stimulation is often described in terms of total charge delivered by a stimulation pulse. By definition, charge is the product of the magnitude of stimulating current and the duration of the stimulus pulse. Given the limits on safe injectable charge imposed by the electrochemical properties of the electrode, it is important to optimize the impact of current magnitude and pulse width for stimulation efficacy. In this work, we define efficacy as the ability to produce ganglion cell responses in response to electrical stimulation. It is commonly understood that there may be multiple aspects of ganglion cell responses that may be important for different aspects of prosthetic vision restoration. Here, we largely focus on two measurements of electrical stimulation, the stimulation threshold, defined as the current needed to elicit a spike on $50 \%$ of the trials, and the dynamic range, defined as the ratio of the maximal number of spikes evoked within the CIC limit and the spikes evoked at the threshold.

To examine how responses to the same amount of charge differ with different combinations of current and time we used a $30 \mu \mathrm{m}$ diameter electrode to deliver an equivalent level of charge for a combination of stimulus current and duration (Fig. 4). We characterized spiking responses by measuring the total number of spikes elicited as well as the pattern of evoked spikes. The average magnitude of the spike response was significantly greater at each fixed stimulating charge level for a 1 versus a $5 \mathrm{~ms}$ pulse for all charge levels $>5 \mathrm{nC}$ ( $t$ test, $p<0.05 ; n=45$ for both pulse widths). There were nearly twice as many total spikes at the maximum stimulating charge of $25 \mathrm{nC}$ ( $t$ test, $p<0.05$ ); however, only a subset of cells was tested at this charge level out of concern for possibly damaging electrodes by operating at the upper limit of the safe injectable charge $(n=10$ for $1 \mathrm{~ms}$ pulse width and $n=21$ for $5 \mathrm{~ms}$ pulse width). As expected, the responses of RGCs increase with increasing charge for both pulse widths. Overall, for the entire range of safe 

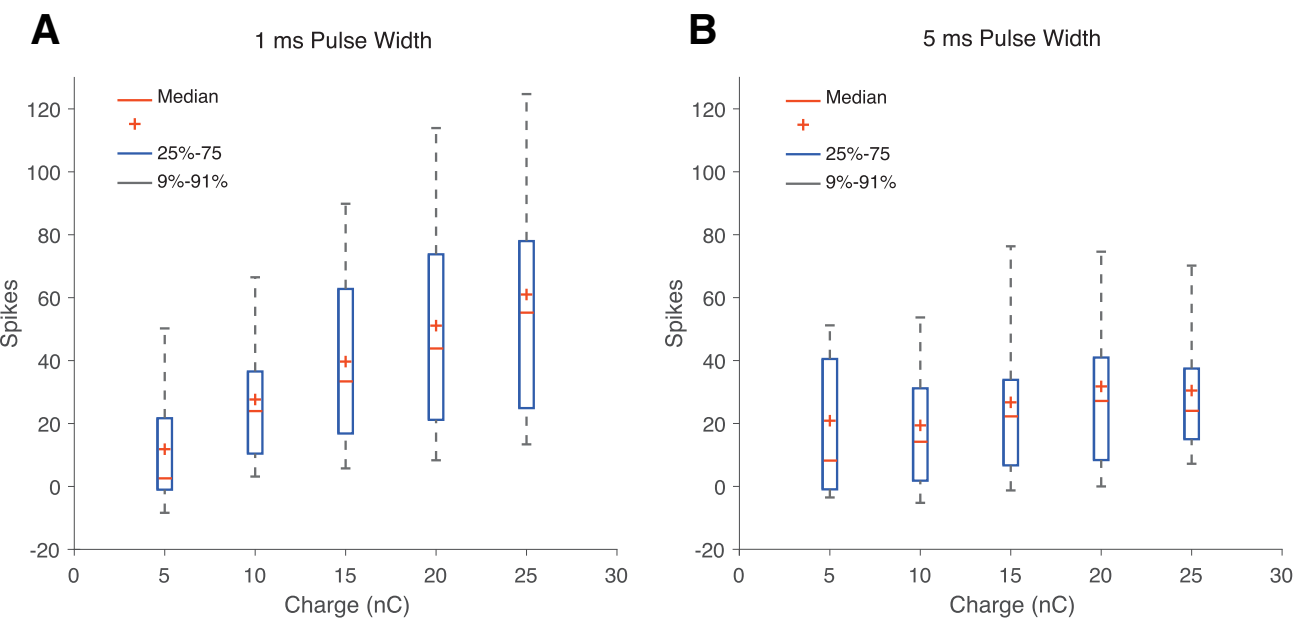

Figure 4. Average response of RGC to stimulation with a $30-\mu \mathrm{m}$ diameter electrode using 1 or 5 ms pulse widths plotted against stimulating charge within $25 \mathrm{nC}$ charge injection limits. $\boldsymbol{A}$, Range of spiking responses for stimuli with a 1 ms pulse width. $\boldsymbol{B}$, Range of spiking responses for stimuli with a $5 \mathrm{~ms}$ pulse width. For both pulse widths, average spikes increase with increasing charge. At the same charge level, shorter pulse widths elicit more spikes (for $1 \mathrm{~ms}, 5$ to $20 \mathrm{nC}$ range, $n=45$; at $25 \mathrm{nC}, n=10$; for 5 ms, $n=21$ ). Red plus denote mean.

injectable charge, the shorter $1 \mathrm{~ms}$ pulse width elicited a greater number of spikes than the longer $5 \mathrm{~ms}$ pulse width (ANOVA, $p<0.001$ ).

To examine differences in the pattern of spikes following stimulation between 1 and $5 \mathrm{~ms}$ pulse widths at equivalent charge, we plotted the peristimulus time histogram for spikes up to $500 \mathrm{~ms}$ after stimulation, across a population of 45 cells. In general, the pattern of evoked short and long latency spikes for both pulse widths appear similar within the same cell, but there is significant variation in spiking behavior between cells over the entire population of observed cells. This can be observed when evaluating the pattern of evoked responses at $20 \mathrm{nC}$ for 1 and $5 \mathrm{~ms}$ pulse widths for all cells (Fig. $5 A, B)$. Comparing the average response at each pulse width at every charge level, there is a greater magnitude of both short latency $(<100 \mathrm{~ms})$ and long latency spikes $(<100 \mathrm{~ms})$ for the $1 \mathrm{~ms}$ pulse duration (Fig. $5 C, D$ ).

\section{Influence of electrode diameter on neural response}

High acuity retinal prostheses require an array with a high density of stimulating electrodes, because electrode spacing should be directly correlated with the resolution of restored vision (Palanker et al., 2005; Moghadam et al., 2013; Yang et al., 2016). Therefore, significant effort has been dedicated to decreasing stimulating electrode area and spacing, however, smaller electrodes have less available CIC. To examine how electrode diameter influences electrical excitation of the retinal circuitry, we compared the effectiveness of 10,20 , and $30 \mu \mathrm{m}$ diameter SIROF electrodes at achieving retinal stimulation within the limits of their respective CIC. Based on the above results, showing that the maximum spike response within the CIC is larger for shorter pulses, we chose to use stimuli with a $1 \mathrm{~ms}$ pulse width for this comparison. Because repetitive trains of stimulation pulses may likely be used to encode temporal properties of vision in retinal prosthetics as opposed to single pulses in isolation, we also examined responses to pulse trains of 20 simulation pulses at $500 \mathrm{~Hz}$ (Lee et al., 2013; Im and Fried, 2016).
We were able to evoke responses within the CIC for each electrode diameter with a single stimulation pulse, although the number of cells that reached stimulation, the threshold to evoke spikes, and the number of evoked spikes varied with electrode diameter. While we could evoke stimulation with the $10 \mu \mathrm{m}$ diameter electrodes in some cells, we noticed that fewer cells responded to stimulation with $10 \mu \mathrm{m}$ diameter electrodes than at other diameters.

To explore this observation further, we used a subset of cells which were approximately equidistant to all three sizes of electrodes. This allowed us to compare responses to different size electrodes within the same cell. In cases where electrodes were not exactly equidistant, we minimized the distance between the cell of interest and the smaller electrodes. This resulted in a mean distance to the electrode of $31.8,43.3$, and $80.0 \mu \mathrm{m}$ for 10 , 20 , and $30 \mu \mathrm{m}$ diameter electrodes, respectively, for this within cell electrode comparison. In $53 \%$ of the cells $(n=21 / 40)$, we failed to get stimulation from a $10 \mu \mathrm{m}$ diameter stimulating electrode in a cell that responded to a 20 or $30 \mu \mathrm{m}$ diameter electrode that was equidistant or farther from the $10 \mu \mathrm{m}$ diameter electrode, indicating that $10 \mu \mathrm{m}$ diameter electrode are less effective at recruiting $R G C$ responses than larger diameters. While, it may be expected that smaller electrodes could be less effective at stimulating at longer distance, within this restricted range of the within cell comparison, we found no correlation between distance from cell and likelihood of stimulation.

For all electrode diameters, we found that spike number generally increased monotonically with increasing current for all diameters when averaged across a group of cells, although there was significant cell to cell heterogeneity of evoked responses (Fig. 6). At equivalent stimulating charge, smaller electrodes evoke a greater spiking response. For example, at 5 and $10 \mathrm{nC}$ charge for a single stimulus pulse, a $20 \mu \mathrm{m}$ diameter electrodes evoked $3.1 \times$ ( $t$ test, $p<0.01)$ and $2.4 \times(t$ test, $p<0.01)$ more spikes 
A

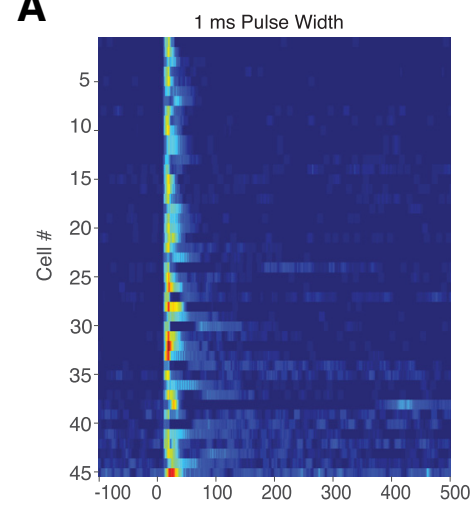

C

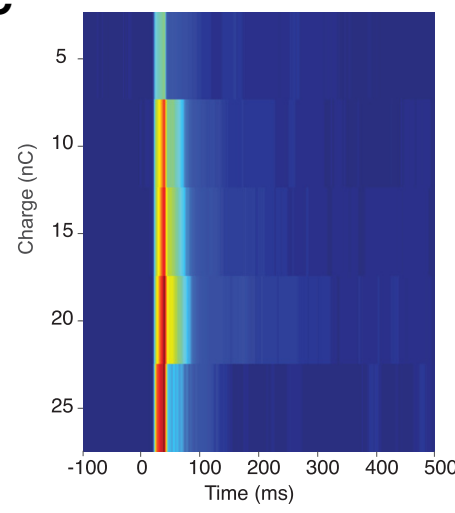

B

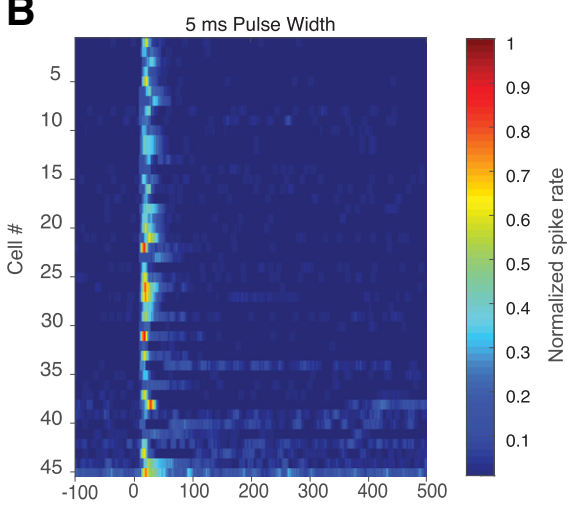

D

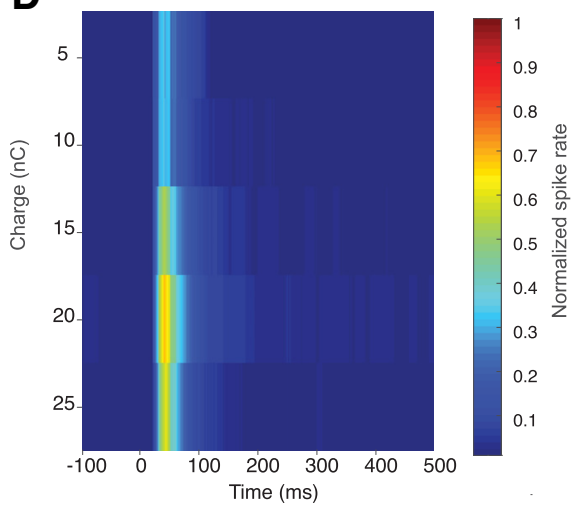

Figure 5. Heat map of normalized spike rates evoked by stimulation using 1 and $5 \mathrm{~ms}$ pulse width using a $30 \mu \mathrm{m}$ iridium oxide electrode for $50 \mathrm{~ms}$ before stimulation to $500 \mathrm{~ms}$ after stimulation. Top row, Comparison of spike magnitude and latency for 45 RGCs stimulated with $20 \mathrm{nC}$ of charge using either $(\boldsymbol{A}) 1 \mathrm{~ms}$ pulse or $(\boldsymbol{B}) 5 \mathrm{~ms}$ pulse widths. Cells are ordered by the magnitude of their response to $1 \mathrm{~ms}$ stimulation between time 0 and $500 \mathrm{~ms}$. Bottom row, Average spike rates for a range of equivalent charge for $1 \mathrm{~ms}$ $(\boldsymbol{C})$ and $5 \mathrm{~ms}(\boldsymbol{D})$ stimuli, for cells in $\boldsymbol{A}, \boldsymbol{B}$. Color scale is normalized spiking to maximal rate in dataset.

than a $30 \mu \mathrm{m}$ diameter electrode, respectively (Fig. 6D,E). These observations also hold true for pulse train stimulation, although more spikes were evoked with pulse trains than single pulse stimulation across all electrode diameters and stimulation charge (Fig. $6 F-H)$.

A key measure used to describe the efficiency of retinal stimulation is charge threshold, defined as the charge level needed to evoke at least one spike on $50 \%$ of the trials. Here, we measured the charge threshold for our different electrode diameters and found that stimulation thresholds increased with electrode size. The threshold charge was $0.83,1.8$, and $3.6 \mathrm{nC}$ for $10 \mu \mathrm{m}(n=19), 20 \mu \mathrm{m}$ $(n=61)$, and $30 \mu \mathrm{m}(n=108)$ diameter electrodes, respectively, using single stimulus pulses (Fig. 7A). Results for pulse train stimulation mirrored the findings for single pulse stimulation with the exception that pulse train stimuli had lower charge thresholds than single pulses (unpaired $t$ test: $p<0.05$ ). For pulse trains, the charge thresholds were $0.46,1.6$, and 2.4 $\mathrm{nC}$ for $10 \mu \mathrm{m}(n=26), 20 \mu \mathrm{m}(n=70)$, and $30 \mu \mathrm{m}(n=62)$ diameter, respectively (Fig. 7A). Taken together, these results indicate that smaller electrodes are generally more efficient at evoking RGC spiking, on a per charge basis, with the important caveat, that $10 \mu \mathrm{m}$ diameter electrodes evoked responses in fewer cells compared with 20 and $30 \mu \mathrm{m}$ diameter electrodes.
While numerous studies have examined stimulation threshold in the past, the absolute range of responses that can be evoked within the usable range of electrical charge has been considered far less. This is an important property to consider for prosthetic stimulation as it ultimately determines the range of visual information that can be encoded and the resolution of continuously varying responses that can be discriminated. If we consider a stimulus that only produces threshold responses (a single spike $50 \%$ of the time), this would probably not reproduce satisfactory vision as it would be a very weak percept to encode a range of input conditions.

Therefore, we quantified the dynamic range as the ratio of the maximal number of spikes evoked within the CIC limit and the spikes evoked at the threshold (Fig. 8A,B). Consistent with our observation that larger electrodes can evoke more spikes within their CIC limit, we found the dynamic range of $R G C$ responses to subretinal stimulation differed over electrode diameter (one-way ANOVA, $p<0.05$ ), and the dynamic range for $10 \mu \mathrm{m}$ diameter electrodes was significantly different compared with compared with 20 or $30 \mu \mathrm{m}$ electrodes for both single stimuli and pulse trains. The dynamic range for a $30 \mu \mathrm{m}$ electrode was $1.6 \times$ and $1.3 \times$ that of $10 \mu \mathrm{m}$ diameter electrodes on the decibel scale, for single and pulse trains stimulation, respectively. 
A

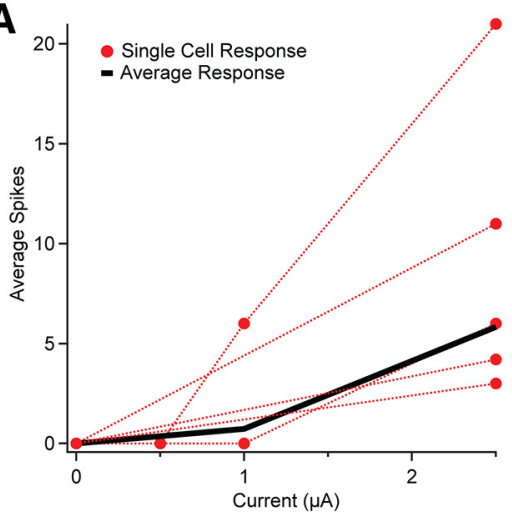

D
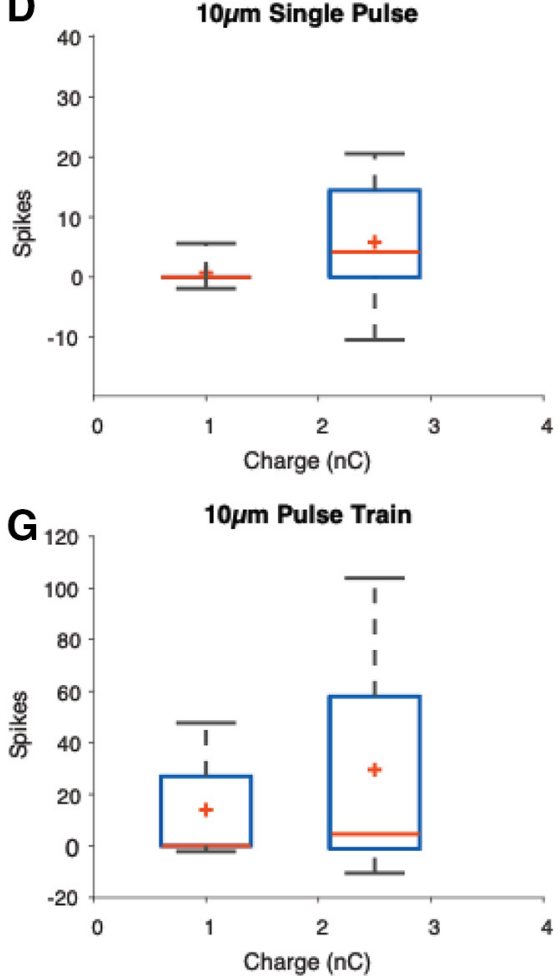

B

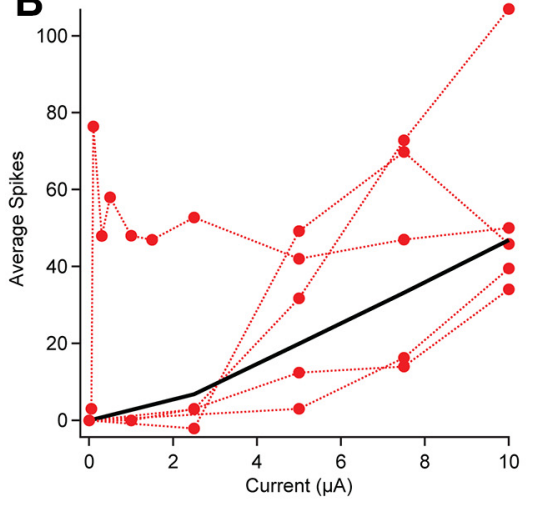

E

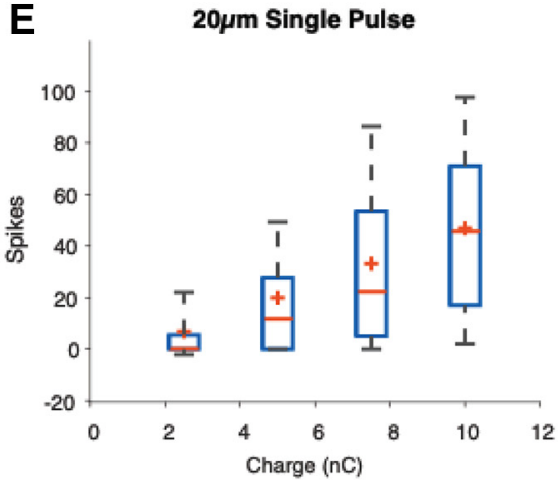

$\mathbf{H}_{1}$

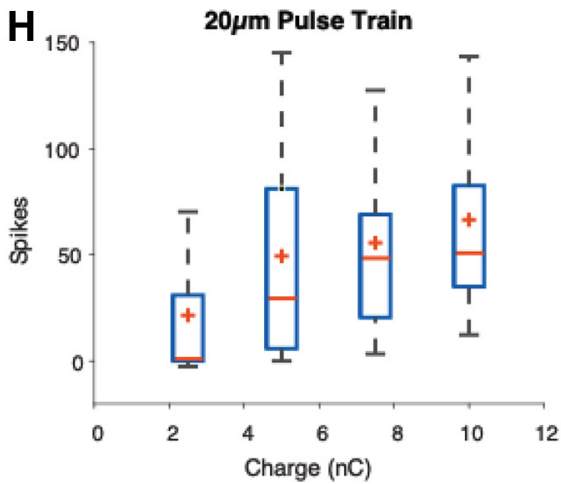

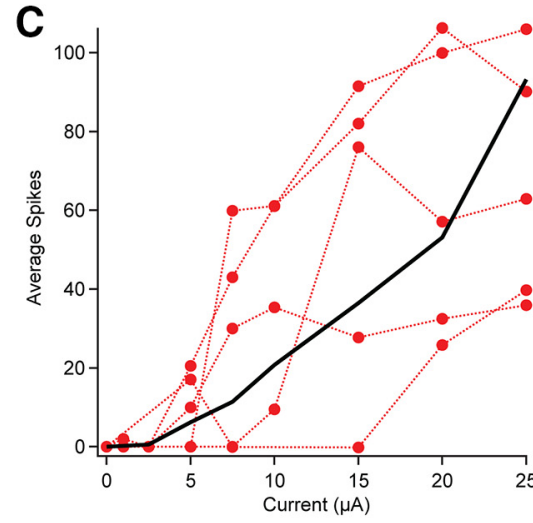

$\mathbf{F}$

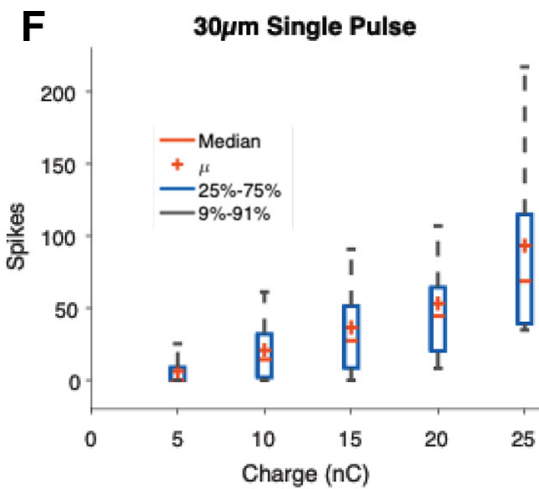

I

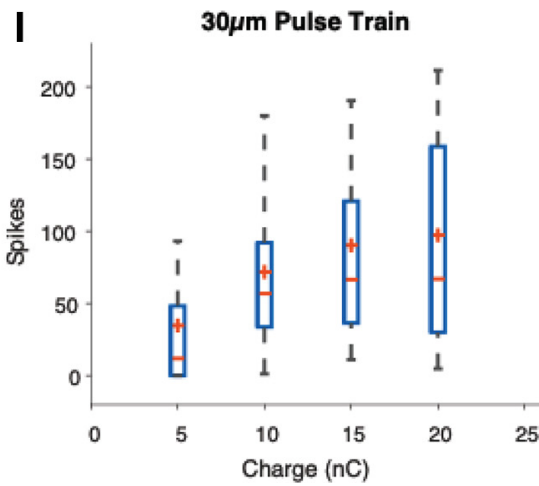

Figure 6. RGC stimulation with $1 \mathrm{~ms}$ pulses for 10,20 , and $30 \mu \mathrm{m}$ electrodes. $\boldsymbol{A}-\boldsymbol{C}$, Stimulus response relationship for five individual example RGCs (red) and average response (black) within the CIC limits of each respective electrode size. $\boldsymbol{C}-\boldsymbol{F}$, Stimulation thresholds for stimulation at each electrode diameter for single pulses and (G-I) pulses trains. Red plus denotes mean.

What could account for these differences in stimulation between electrode sizes? Electrical stimulation of neural tissue occurs when injected current creates an electric field in the extracellular space that encompasses all or part of the neuron. The electric field causes a redistribution of charge along the cell surface, altering transmembrane voltage and leading to the activation of voltage-gated ion channels that trigger an action potential or synaptic release. The magnitude of the electric field is proportional to the stimulating charge density. Consequently, charge density has been discussed as a metric to normalize the amount of charge needed for stimulation across different electrode geometries (Palanker et al., 2005), however, when comparing previous studies with varying electrode sizes there is a wide range of results reported for the required charge density for effective stimulation (Stett et al., 2000; Tsai et al., 2009; Yang et al., 2011; Im and Fried, 2015; Corna et al., 2018). Importantly, Corna et al. (2018) demonstrated that stimulation threshold expressed in charge density is not conserved for variety of total electrode areas, when simulating large electrodes by grouping $30 \mu \mathrm{m}$ diameter electrodes. For epi-retinal stimulation, Sekirnjak et al. (2006) also found charge density was not conserved across a range of electrode areas.

To examine this question for subretinal stimulation at relevant electrode diameters, we converted charge thresholds to charge density thresholds based on the geometric area (Fig. 9A,B). Charge thresholds significantly differed across electrode diameters (one-way 
A

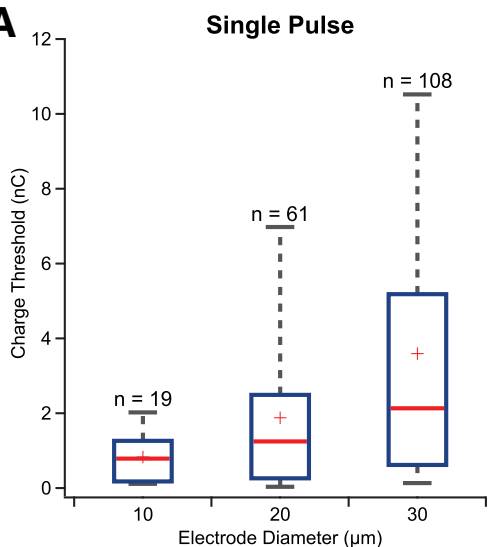

B

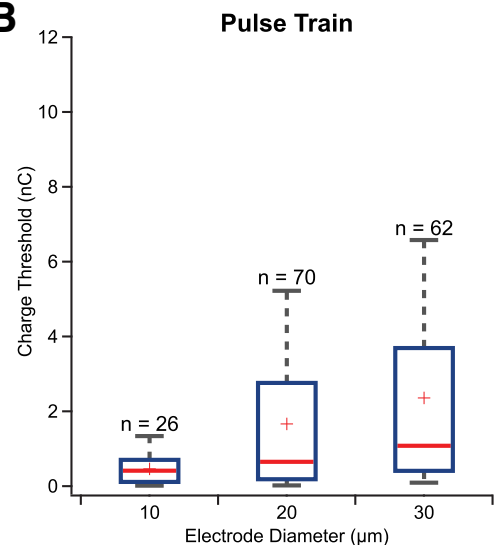

Figure 7. Comparison of 10,20 , and $30 \mu \mathrm{m}$ responses: $(\boldsymbol{A})$ charge threshold for stimulation for single pulses and $(\boldsymbol{B})$ pulse trains. Blue box denotes $25 \%-75 \%$ range. Gray whiskers denotes 9\%-91\% range. Red line denotes median, and red plus denotes mean.

ANOVA, $p<0.05$ ). In contrast to charge thresholds, we observed the highest charge density thresholds for the smallest electrodes and a decreasing trend in charge density thresholds with increasing electrode area, for both single stimuli and pulse trains, with a significant interaction between 10 and $30 \mu \mathrm{m}$ diameter electrodes.

As shown above, threshold is not the only difference between electrode diameters; the absolute range of responses evoked over a range of stimulation currents is another important metric that differs between electrode sizes. To understand how charge density might contribute to the differences in stimulation efficiency across a range of stimulation levels, we plotted spiking response against charge density for our different electrode diameters (Fig. $9 C, D)$. At equivalent charge density, the spiking response measured for RGCs differs strongly between electrode diameters. Larger diameter electrodes are more effective at eliciting spikes for both single pulses and pulse trains, even when the charge density is equal. These data suggest that stimulation efficacy is not well explained by charge density, even for electrode geometries that are relatively similar, such as our planar electrodes ranging from
10 to $30 \mu \mathrm{m}$ diameter. Specifically, this allows us to reject the hypothesis that charge density alone determines neural stimulation over a relevant range of electrode areas.

What could account for differing stimulation efficiencies at the same charge density and electric field strength? Given that electric field strength is non-uniform and decays with distance from the electrode, the location of a neuron within the electric field is an important consideration in determining the efficacy of stimulation, particularly for electrodes on the scale of individual neurons. We hypothesized that differences in the size of the electric field evoked by different size electrodes may account for differences in stimulation efficiency with electrode size, even when charge density is constant.

To test this idea, we modeled the electric field using a 3-D finite element model for each electrode diameter. For a fixed current density corresponding to the maximum injectable charge at each electrode diameter in a $1 \mathrm{~ms}$ pulse width and assuming $1000 \mathrm{~V} / \mathrm{m}$ as a minimum electric field to induce stimulation (Moghadam et al., 2013). Visualizing the magnitude and spread of the electric field in the $y$ and $z$ dimensions reveals that the spread of the
A

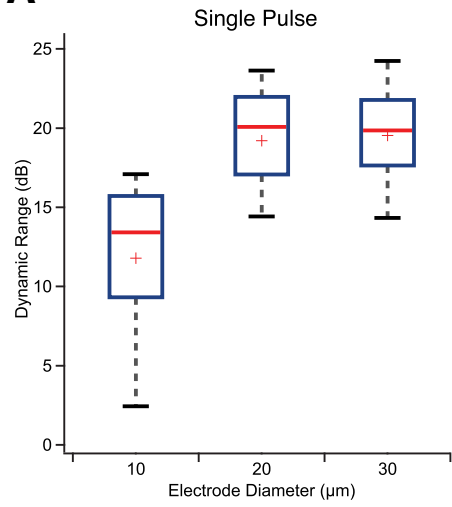

B

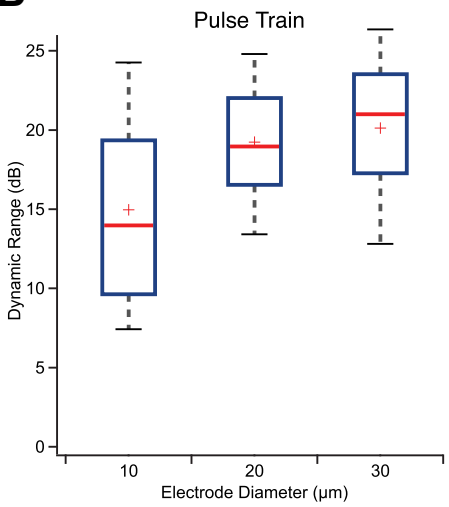

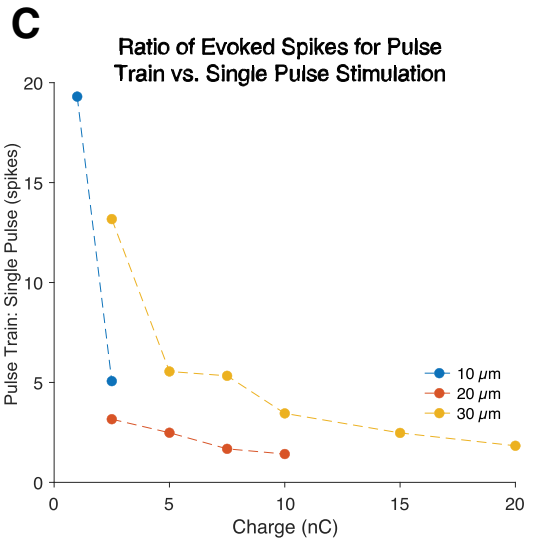

Figure 8. Comparison of available dynamic range of responses for 10,20 , and $30 \mu \mathrm{m}$ diameter electrodes for $(\boldsymbol{A})$ single pulses, and $(\boldsymbol{B})$ pulse trains, expressed in decibels. Blue box denotes 25\%-75\% range. Gray whiskers denotes $9 \%-91 \%$ range. Red line denotes median, and red plus denotes mean. $\boldsymbol{C}$, Comparison of retinal stimulation efficacy with pulse trains versus a single pulse stimulus. Ratio of spikes evoked for pulse train to single pulse stimuli at equivalent stimulating charge levels for each stimulating electrode diameter. 
A

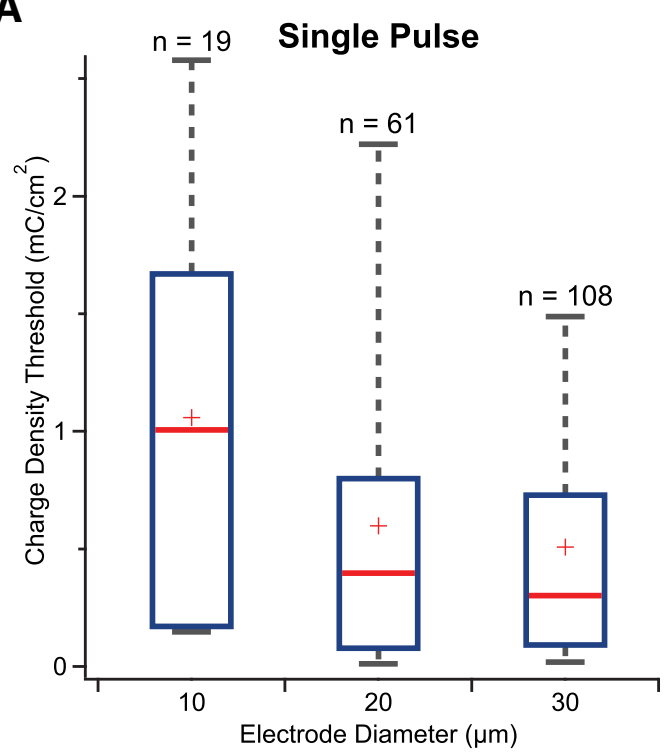

C

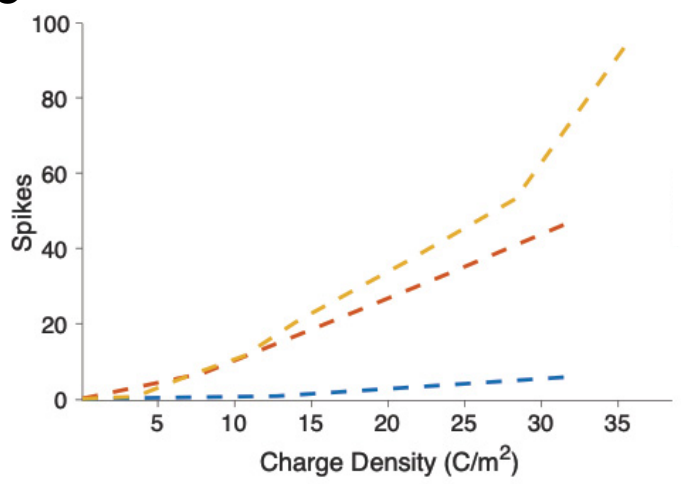

B

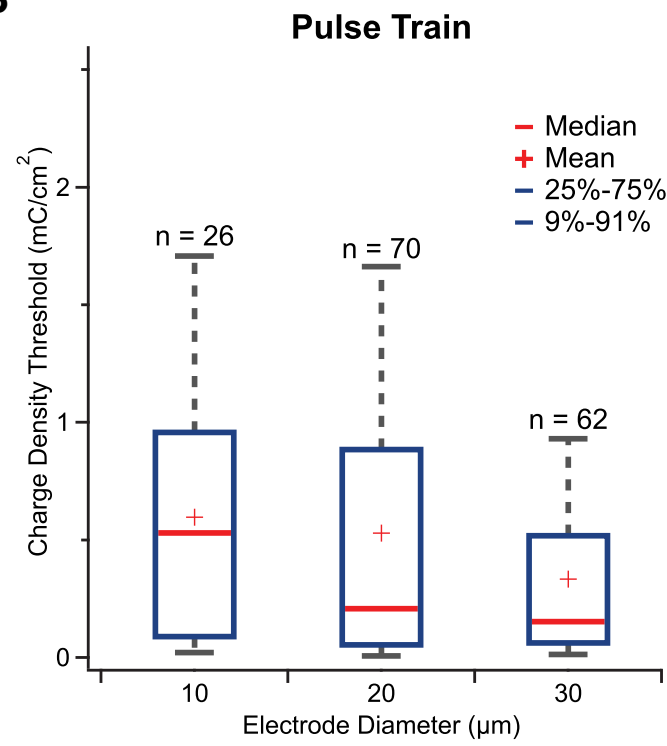

D

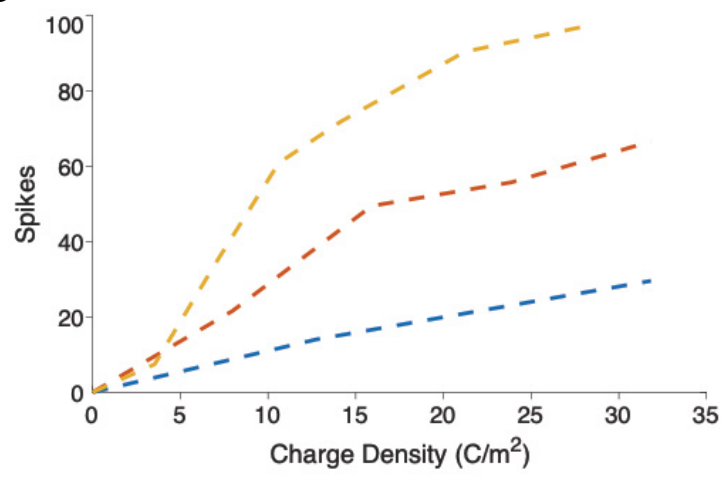

$--10 \mu \mathrm{m} \quad--20 \mu \mathrm{m} \quad--30 \mu \mathrm{m}$

Figure 9. Charge density thresholds for 10, 20, and $30 \mu \mathrm{m}$ electrodes. $\boldsymbol{A}$, Charge density thresholds for single pulses $(\boldsymbol{B})$ and pulse trains. $\mathbf{C}$, Intensity response relationship for evoked spikes versus stimulating charge density for single pulses $(\boldsymbol{D})$ and pulse trains.

stimulating electric field depends strongly on electrode size (Fig. 10A). Within the limits of the electrode CIC, the effective stimulation depth of a $10 \mu \mathrm{m}$ diameter electrode for a $1 \mathrm{~ms}$ pulse width and $2.5 \mu \mathrm{A}$ stimulation current is limited to $a \sim 20 \mu \mathrm{m}$ radius from the surface of the electrode. The modeled stimulation depth of 20 and $30 \mu \mathrm{m}$ electrodes extends much further to a $35 \mu \mathrm{m}$ and $55 \mu \mathrm{m}$ radius, respectively. The short depth of effective stimulation may account for the poor reliability of retinal stimulation with $10 \mu \mathrm{m}(47 \%)$ electrodes versus 20 and $30 \mu \mathrm{m}$ electrodes $(100 \%)$ and supports our hypothesis that the difference in the stimulation efficacy between electrode sizes can be attributed to the penetration depth and/or spread of the electric field. The electric field shape over the scale of target neurons provides a framework for understanding differences in stimulation efficiency between different electrodes sizes when current density is held constant. Importantly, the penetration depth of electric field within the extent of safe injectable charge limits suggests a significant limitation on electrode scaling for subretinal prostheses (Fig. 10B).

\section{Discussion}

Development of high-acuity retinal prosthetics may enable treatment of common forms of blindness caused by retinal degeneration. Advances in microfabrication and the development of high $\mathrm{CIC}$ electrode materials have enabled the development of high-resolution (>20/400 acuity) retinal prosthetics; however, there remains a poor understanding of the constraints placed on neural stimulation by electrodes at this small scale. Here, we characterized the neural responses to high density retinal prosthetic stimulation electrodes made of sputtered iridium oxide (SIROF) with 10,20 , and $30 \mu \mathrm{m}$ diameter. Importantly, we first characterized the charge injection limits to only examine responses within their electrochemical capabilities. Here, we identified key aspects of neural stimulation that are constrained by these electrochemical limitations. 

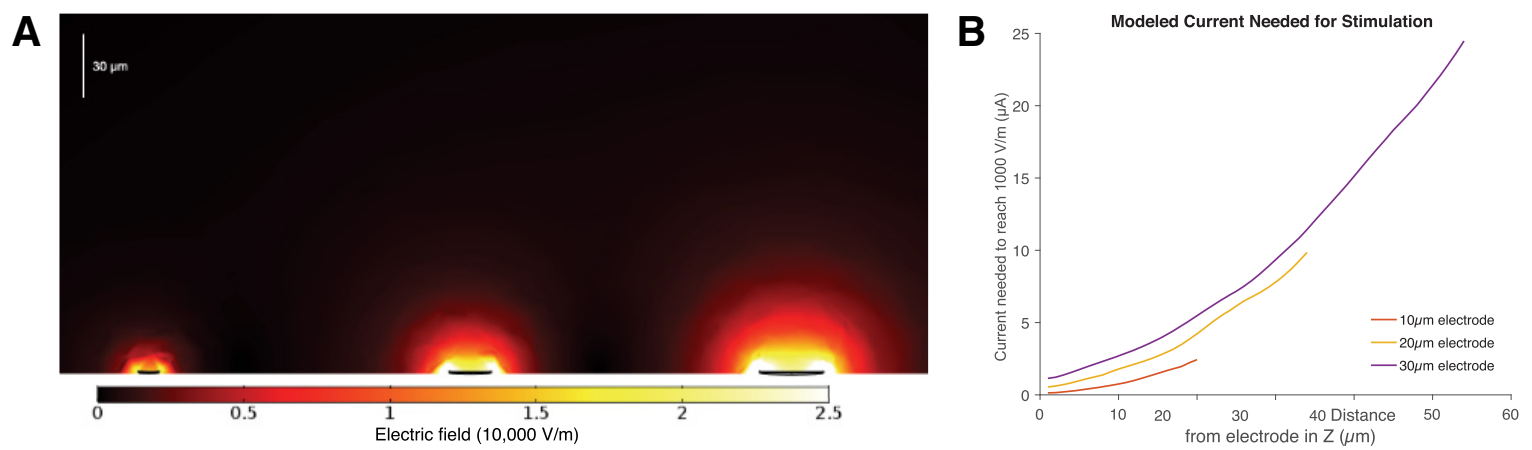

Figure 10. Modeling penetration depth of stimulating electric field for different iridium oxide electrode diameters. $\boldsymbol{A}$, Cross section ( $Y-Z$ plane) heatmap visualization of the electric field induced by the injection of current from a 10, 20, and $30 \mu \mathrm{m}$ (left to right) electrode at charge injection limits for a $1 \mathrm{~ms}$ pulse in a 3-D finite element model of retinal tissue. The penetration depth and magnitude of electric field are directly related to the size of the electrode and injected current. $\boldsymbol{B}$, Calculation of the stimulation current needed to induce a $1000 \mathrm{~V} / \mathrm{m}$ electric field at a $z$-height above the stimulating electrode. The required current curves for each diameter terminate at the respective maximum current for a $1 \mathrm{~ms}$ pulse at the charge injection limit.

Electrical stimulation of retina has been studied over many decades. Much of the fundamental electrophysiological characterization of retinal responses to intracellular or extracellular stimulation has been done using glass micro-pipettes embedded within the retina to deliver charge. While this can create highly focal electric fields, the experimental parameter space is not bounded by practical limits on current, charge, geometry, or electrode material. Other studies have used more realistic geometry with planar metal electrodes (Stett et al., 2000, 2007; Yang et al., 2011; Cai et al., 2013; Sim et al., 2014; Im and Fried, 2015; Stutzki et al., 2016; Ho et al., 2019). While, many of these studies characterized stimulation efficacy in healthy retina with an intact photoreceptor cell layer, which significantly affects stimulation thresholds (Stett et al., 2000, 2007; Boinagrov et al., 2014; Sim et al., 2014). Few studies have systematically explored the range of responses that can be evoked above stimulation threshold or the relationship to electrode size/geometry. In addition, there have been extensive theoretical (Palanker et al., 2005) and experimental efforts (for review, see Sekirnjak et al., 2006; Corna et al., 2018), however, as these reviews of experimental results reveal there is considerable variability in the published results, leaving much uncertainty about the specific physical considerations that drive stimulation efficacy. Importantly, charge injection limits have rarely been considered beyond a brief mention of the reported CSC for the electrode material. To the best of our knowledge, this is the first report of dynamic range for electrodes of this scale within the specific charge injection limits of SIROF electrodes.

Working within the CIC limits of SIROF electrodes of different sizes, we evaluated the effectiveness of stimulation at each electrode size in terms of stimulation thresholds and the dynamic range of stimulation. Importantly, we found that stimulation thresholds occur at $38 \%(10 \mu \mathrm{m})$, $23 \%(20 \mu \mathrm{m})$, and $18 \%(30 \mu \mathrm{m})$ of the maximum available charge within the $\mathrm{CIC}$. Although the thresholds were within the $\mathrm{CIC}$ for all diameters, maximal responses were limited by the $\mathrm{CIC}$ of the electrode rather than the physiology and indicate that the dynamic range of responses is strongly influenced by electrode size, a novel finding that has not been appreciated by previous studies focused on stimulation thresholds. Importantly, of those cells that were responsive to stimulation with a $30 \mu \mathrm{m}$ electrode, $<50 \%$ could be stimulated with a $10 \mu \mathrm{m}$ electrode that was as close or close to the cell, indicating that $10 \mu \mathrm{m}$ diameter electrodes are less reliable at evoking responses than larger electrodes. These limitations on dynamic range and efficiency have important implications for retinal prosthetic designs.

In addition, this work also revealed how current magnitude and total charge relate to stimulation efficiency and dynamic range of responses. We found that the absolute current magnitude of the delivered charge has a significant influence on retinal stimulation efficacy. Past work has often focused on total charge delivered or charge density. In contrast, we show here that neither of these properties fully account for stimulation efficiency. We find that shorter duration, higher current magnitude stimulation parameters evoked more spikes than longer pulses of a lower current magnitude.

Past studies of subretinal stimulation have identified that short latency responses $(<5-10 \mathrm{~ms})$ are characteristic of direct RGC stimulation and longer latency responses $(>10 \mathrm{~ms})$ can be attributed to indirect network mediated stimulation (Jensen et al., 2005; Sekirnjak et al., 2006; Margalit et al., 2011; Sim et al., 2014). Notably, some previous studies have proposed that longer pulse widths yield greater selectivity for indirect stimulation (Margalit et al., 2011; Boinagrov et al., 2014). Although we have not attempted to assign any functional designation to the different spike latencies here, our results indicate that this must hold true only at a fixed current level where a longer pulse duration results in more delivered charge. Here, we show that greater current is more effective at evoking both short and long latency spikes for an equivalent charge.

These findings have important implications for the design of high acuity retinal prostheses. Charge density is often considered as the relevant parameter for comparing neural stimulation across different electrode scales. Here, we find that larger electrodes, with a capacity to deliver greater current, are more effective at evoking RGC responses than smaller electrodes, even at the same 
current density. These results consistent with recent work by Corna et al. (2018), where they found that larger electrode areas used by grouping multiple electrodes together had lower charge density thresholds and were more effective at evoking spikes at equivalent charge density. This indicates that when designing hardware to deliver current, it is important to consider the maximum range of spikes that can be evoked based on the limits of the current source. For retinal implants that can only source a limited or fixed current output at each electrode, longer pulse widths may be required. In contrast, for implants that can supply a wider current range, a short pulse width and current injection near the limit of the CIC may achieve the broadest range of stimulation responses and is more efficient, yielding the most spikes for a given amount of charge. Given, that $10 \mu \mathrm{m}$ diameter electrodes failed to evoke responses in a majority of ganglion cells that responded to larger diameter electrodes, there may be a practical limit for miniaturizing the scale of planar iridium oxide electrodes used for effective retinal stimulation. Our results from electric field modeling indicate that this limit is related to the size of the electric field relative to target neurons. Consequently, this limitation on minimum electrode size also limits the possible spatial resolution of an implanted electrode array.

The limited dynamic range of RGC responses within the safe injectable charge limits at all diameters indicates a need for better electrode materials at small electrodes scales. In general, the number of evoked spikes increases linearly with increasing current and does not saturate within the limits of $\mathrm{ClC}$ for the electrode sizes tested here. Alternative electrode materials such as conductive polymers including poly 3,4-ethylenedioxythiophene (PEDOT) or nanostructured platinum or iridium may enable higher charge injection and improve the efficacy of stimulation at $10 \mu \mathrm{m}$ electrode size or smaller (Ganji et al., 2017, 2019). Additionally, high acuity retinal prostheses may require 3$D$ electrodes that penetrate through the retina to reduce electrode-tissue separation and thereby improve the effectiveness of stimulation at small electrode scales (Ganesan et al., 2010; Flores et al., 2018).

There are several caveats to consider with regards to this experimental approach and scope of our results. The stimulus pulse duration did not extend past $5 \mathrm{~ms}$ because of hardware restrictions of the current source. Longer pulse durations extending up to $50 \mathrm{~ms}$ and proportionally lower current may yield further insights into the strengthduration characteristics of retinal stimulation within $\mathrm{CIC}$ limits, though our results here suggest that longer pulses are less efficient on a per charge basis. Also, the stimuli tested here consist of single pulses or $20 \times$ pulse trains at $500 \mathrm{~Hz}$. While using pulse trains may be an effective strategy to reduce stimulation threshold and increase the dynamic range of spikes, the responses began to saturate at the maximum charge injection limit, indicating a diminishing impact of repetitive stimulation. Further characterization using subretinal stimuli at varying frequency and duty cycle may yield greater insight into the possible range of evoked responses that can encode functional visual information within $\mathrm{CIC}$ limits.
Throughout this work, we have averaged across a heterogeneous population of ganglion cell types. Indeed, our data showed a diversity of the range and latency of spiking responses, a relatively broad distribution of current thresholds, and different current/spiking functions. We feel justified in using the mean response of the population, because currently there is insufficient data to suggest the possibility of selective targeting of any particular population of ganglion cells (but see Fan et al., 2019). Therefore, a general goal of retinal prosthetic stimulation is to recruit activity in the largest population of ganglion cells. For an actual prosthesis, the retina receives concurrent stimulation from an array of electrodes resulting in a complex pattern of evoked responses across diverse cell types. Although we have characterized responses only from a single cell using a single stimulating electrode, this a necessary foundational step to determining the minimum size at which each electrode is independently effective within a dense array.

Ultimately, the relationship between injected charge and neural stimulation results from complex interactions that depend on the shape and magnitude of the electric field, the geometry of neural processes traversing the electric field, and the complement of voltage-gated ion channels in the target neurons. Consequently, a universally scalable relationship between the magnitude of charge delivered through stimulating electrodes and the resulting effectiveness of retinal stimulation will be complex, however, this approach of characterizing evoked responses within quantified $\mathrm{CIC}$ limits can help establish a framework to compare between or develop new microelectrodes for high visual acuity subretinal prosthesis.

\section{References}

Boinagrov D, Pangratz-Fuehrer S, Goetz G, Palanker D (2014) Selectivity of direct and network-mediated stimulation of the retinal ganglion cells with epi-, sub- and intraretinal electrodes. J Neural Eng 11:026008.

Boinagrov D, Lei X, Goetz G, Kamins TI, Mathieson K, Galambos L, Harris JS, Palanker D (2016) Photovoltaic pixels for neural stimulation: circuit models and performance. IEEE Trans Biomed Circuits Syst 10:85-97.

Bourne RRA, Flaxman SR, Braithwaite T, Cicinelli MV, Das A, Jonas JB, Keeffe J, Kempen JH, Leasher J, Limburg H, Naidoo K, Pesudovs K, Resnikoff S, Silvester A, Stevens GA, Tahhan N, Wong TY, Taylor HR, Bourne R, Ackland P, et al. (2017) Magnitude, temporal trends, and projections of the global prevalence of blindness and distance and near vision impairment: a systematic review and meta-analysis. Lancet Glob Heal 5:e888-e897.

Cai C, Twyford P, Fried S (2013) The response of retinal neurons to high-frequency stimulation. J Neural Eng 10:036009.

Cogan SF (2008) Neural stimulation and recording electrodes. Annu Rev Biomed Eng 10:275-309.

Cogan SF, Ehrlich J, Plante TD, Smirnov A, Shire DB, Gingerich M, Rizzo JF (2009) Sputtered iridium oxide films for neural stimulation electrodes. J Biomed Mater Res B Appl Biomater 2004:41534156.

Corna A, Herrmann T, Zeck G (2018) Electrode-size dependent thresholds in subretinal neuroprosthetic stimulation. J Neural Eng 15:045003.

Damle S, Liu YH, Arya S, Oesch NW, Lo YH (2020) Vertically integrated photo junction-field-effect transistor pixels for retinal prosthesis. Biomed Opt Express 11:55-67. 
Fan VH, Grosberg LE, Madugula SS, Hottowy P, Dabrowski W, Sher A, Litke AM, Chichilnisky EJ (2019) Epiretinal stimulation with local returns enhances selectivity at cellular resolution. J Neural Eng 16: e025001.

Flores T, Lei X, Huang T, Lorach H, Dalal R, Galambos L, Kamins T, Mathieson K, Palanker D (2018) Optimization of pillar electrodes in subretinal prosthesis for enhanced proximity to target neurons. $\mathrm{J}$ Neural Eng 15:aaac39.

Ganesan K, Stacey A, Meffin H, Lichter S, Greferath U, Fletcher EL, Prawer S (2010) Diamond penetrating electrode array for epi-retinal prosthesis. Annu Int Conf IEEE Eng Med Biol Soc 2010:6757-6760.

Ganji M, Tanaka A, Gilja V, Halgren E, Dayeh SA (2017) Scaling effects on the electrochemical stimulation performance of $\mathrm{Au}, \mathrm{Pt}$, and PEDOT: PSS electrocorticography arrays. Adv Funct Mater 27:1703019.

Ganji M, Paulk AC, Yang JC, Vahidi NW, Lee SH, Liu R, Hossain L, Arneodo EM, Thunemann M, Shigyo M, Tanaka A, Ryu SB, Lee SW, Tchoe Y, Marsala M, Devor A, Cleary DR, Martin JR, Oh H, Gilja V, et al. (2019) Selective formation of porous Pt nanorods for highly electrochemically efficient neural electrode interfaces. Nano Lett 19:6244-6254.

Goetz GA, Palanker DV (2016) Electronic approaches to restoration of sight. Rep Prog Phys 79:e096701.

Ha S, Khraiche ML, Akinin A, Jing Y, Damle S, Kuang Y, Bauchner S, Lo YH, Freeman WR, Silva GA, Cauwenberghs G (2016) Towards high-resolution retinal prostheses with direct optical addressing and inductive telemetry. J Neural Eng 13:e056008.

Haas J, Rudorf R, Becker M, Daschner R, Drzyzga A, Burkhardt C, Stett A (2020) Sputtered iridium oxide as electrode material for subretinal stimulation. Sens Mater 32:2903-2918.

Hartong DT, Berson EL, Dryja TP (2006) Retinitis pigmentosa Prevalence and inheritance patterns. Lancet 368:1795-1809.

Ho E, Lei X, Flores T, Lorach H, Huang T, Galambos L, Kamins T, Harris J, Mathieson K, Palanker D (2019) Characteristics of prosthetic vision in rats with subretinal flat and pillar electrode arrays. $J$ Neural Eng 16:e066027.

Humayun MS, Dorn JD, Da Cruz L, Dagnelie G, Sahel JA, Stanga PE, Cideciyan AV, Duncan JL, Eliott D, Filley E, Ho AC, Santos A, Safran AB, Arditi A, Del Priore LV, Greenberg RJ (2012) Interim results from the international trial of second sight's visual prosthesis. Ophthalmology 119:779-788.

Im M, Fried SI (2015) Indirect activation elicits strong correlations between light and electrical responses in ON but not OFF retinal ganglion cells. J Physiol 593:3577-3596.

Im M, Fried SI (2016) Temporal properties of network-mediated responses to repetitive stimuli are dependent upon retinal ganglion cell type. J Neural Eng 13:025002.

Jensen RJ, Ziv OR, Rizzo JF (2005) Responses of rabbit retinal ganglion cells to electrical stimulation with an epiretinal electrode. $J$ Neural Eng 2:S16-S21.

Jones BW, Pfeiffer RL, Ferrell WD, Watt CB, Marmor M, Marc RE (2016) Retinal remodeling in human retinitis pigmentosa. Exp Eye Res 150:149-165.

Lee SW, Eddington DK, Fried SI (2013) Responses to pulsatile subretinal electric stimulation: effects of amplitude and duration. J Neurophysiol 109:1954-1968.

Lorach H, Goetz G, Smith R, Lei X, Mandel Y, Kamins T, Mathieson K, Huie P, Harris J, Sher A, Palanker D (2015) Photovoltaic restoration of sight with high visual acuity. Nat Med 21:476-482.

Margalit E, Babai N, Luo J, Thoreson WB (2011) Inner and outer retinal mechanisms engaged by epiretinal stimulation in normal and rd mice. Vis Neurosci 28:145-154.

Moghadam G, Wilke R, Suaning GJ, Lovell NH, Dokos S (2013) Quasi-monopolar stimulation: a novel electrode design configuration for performance optimization of a retinal neuroprosthesis. PLoS One 8:e73130.
Negi S, Bhandari R, Solzbacher F (2012) Morphology and electrochemical properties of activated and sputtered iridium oxide films for functional electrostimulation. J Sens Technol 2:138-147.

Palanker D, Vankov A, Huie P, Baccus S (2005) Design of a high-resolution optoelectronic retinal prosthesis. J Neural Eng 2:S105-S120.

Palanker D, Le Mer Y, Mohand-Said S, Muqit M, Sahel JA (2020) Photovoltaic restoration of central vision in atrophic age-related macular degeneration. Ophthalmology 127:1097-1104.

Pennington KL, DeAngelis MM (2016) Epidemiology of age-related macular degeneration (AMD): associations with cardiovascular disease phenotypes and lipid factors. Eye Vis 3:1-20.

Sekirnjak C, Hottowy P, Sher A, Dabrowski W, Litke AM, Chichilnisky EJ (2006) Electrical stimulation of mammalian retinal ganglion cells with multielectrode arrays. J Neurophysiol 95:3311-3327.

Shire DB, Kelly SK, Drohan WA, Chen J, Doyle P, Gingerich MD, Gingerich MD, Cogan SF, Mendoza O, Wyatt JL, Theogarajan L, Rizzo J, Rizzo J (2009) Development and implantation of a minimally invasive wireless subretinal neurostimulator. IEEE Trans Biomed Eng 56:2502-2511.

Sim SL, Szalewski RJ, Johnson LJ, Akah LE, Shoemaker LE, Thoreson WB, Margalit E (2014) Simultaneous recording of mouse retinal ganglion cells during epiretinal or subretinal stimulation. Vision Res 101:41-50.

Stett A, Barth W, Weiss S, Haemmerle H, Zrenner E (2000) Electrical multisite stimulation of the isolated chicken retina. Vision Res 40:1785-1795.

Stett A, Mai A, Herrmann T (2007) Retinal charge sensitivity and spatial discrimination obtainable by subretinal implants: key lessons learned from isolated chicken retina. J Neural Eng 4:S7-S16.

Stingl K, Bartz-Schmidt KU, Besch D, Chee CK, Cottriall CL, Gekeler F, Groppe M, Jackson TL, MacLaren RE, Koitschev A, Kusnyerik A, Neffendorf J, Nemeth J, Naeem MAN, Peters T, Ramsden JD, Sachs H, Simpson A, Singh MS, Wilhelm B, et al. (2015) Subretinal visual implant alpha IMS - clinical trial interim report. Vision Res 111:149-160.

Stutzki H, Helmhold F, Eickenscheidt M, Zeck G (2016) Subretinal electrical stimulation reveals intact network activity in the blind mouse retina. J Neurophysiol 116:1684-1693.

Tsai D, Morley JW, Suaning GJ, Lovell NH (2009) Direct activation and temporal response properties of rabbit retinal ganglion cells following subretinal stimulation. J Neurophysiol 102:2982-2993.

Wang B, Weiland JD (2015) Resistivity profiles of wild-type, rd1, and rd10 mouse retina. Annu Int Conf IEEE Eng Med Biol Soc 2015:1650-1653.

Wilke RGH, Moghadam GK, Lovell NH, Suaning GJ, Dokos S (2011) Electric crosstalk impairs spatial resolution of multi-electrode arrays in retinal implants. J Neural Eng 8:e046016.

Wong WL, Su X, Li X, Cheung CMG, Klein R, Cheng CY, Wong TY (2014) Global prevalence of age-related macular degeneration and disease burden projection for 2020 and 2040: a systematic review and meta-analysis. Lancet Glob Heal 2:e106-e116.

Yang CY, Tsai D, Guo T, Dokos S, Suaning GJ, Morley JW, Lovell NH (2018) Differential electrical responses in retinal ganglion cell subtypes: effects of synaptic blockade and stimulating electrode location. J Neural Eng 15:046020.

Yang F, Chang MY, Yang CH, Teng CC, Fan LS (2016) Flexible, highdensity microphotodiode array with integrated sputtered iridium oxide electrodes for retinal stimulation. J Micro Nanolithogr MEMS MOEMS 15:e015002.

Yang YT, Lin PK, Wan C, Yang WC, Lin LJ, Wu CY, Chiao CC (2011) Responses of rabbit retinal ganglion cells to subretinal electrical stimulation using a silicon-based microphotodiode array. Invest Ophthalmol Vis Sci 52:9353-9361.

Yue L, Weiland JD, Roska B, Humayun MS (2016) Retinal stimulation strategies to restore vision: fundamentals and systems. Prog Retin Eye Res 53:21-47. 